\title{
Cesárea en el Hospital San Ignacio
}

\author{
Dr. Víctor Rodríguez Romero*
}

\section{INTRODUCCION}

La elección del tema del presente trabajo Cesárea en el Hospital San Ignacio 1983-1984, se debió a dos razones: $\mathrm{La}$ primera, porque se sabe que la revisión de la cesárea, es un método de valor para evaluar la calidad de la atención médica y que suministra variada información sobre la patología predominante, la tendencia agresiva o conservadora de las conductas $y$, finalmente, el conocer la morbimortalidad materna y perinatal.

Por otra parte existe un interés generalizado en analizar el gran incremento que ha tenido la cesárea en las dos últimas décadas y la necesidad de determinar, si los efectos sobre morbimortalidad materna y de recién nacidos confirman el beneficio de dicha tendencia o si por el contrario se concluye que se está abusando de la conducta quirúrgica (21), (24).

En el departamento se ha presentado un incremento importante en el índice de cesáreas en la última década, debido fundamentalmente al cambio de manejo de algunas complicaciones del embarazo, especialmente relacionadas con la distocia, el antecedente de cesárea, la presentación de pelvis, el embarazo múltiple, la ruptura prematura de membranas, y el manejo de la hipertensión $(2,10,17)$. (Ver gráfica No. 1).
GRAFICA No. 1

CESAREA EN EL

HOSPITAL SAN IGNACIO

$1975-1984$

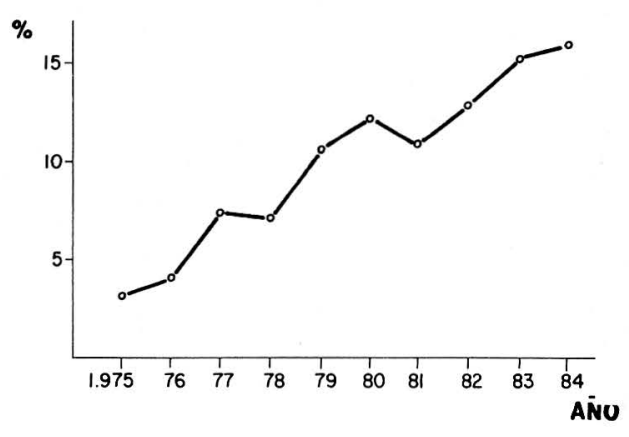

La frecuencia de cesárea se incrementó de $3.1 \%$ en 1975 a $15.3 \%$ en 1984 . Los principales cambios se pueden resumir así:

A. En el departamento se ha abolido prácticamente el uso de fórceps medios y existe la tendencia a resolver con cesárea temprana las distocias, cuando se han agotado otros recursos.

B. La ruptura prematura prolongada de membranas tan frecuente, se maneja en forma agresiva y si las condiciones del cuello no son favorables o falla la inducción, se practica la cesárea. 
C. Para evitar la ruptura uterina y sus graves consecuencias, se emplea la cesárea ante el antecedente de cesárea anterior o miomectomía.

Hasta el año 1979 los casos de antecedentes de cesárea se estudiaban cuidadosamente y según esa evaluación a muchas pacientes se les permitía parto vaginal; en un trabajo realizado en el año 1979 "Morbimortalidad en el Antecedente de Cesárea" (12), se encontraron complicaciones graves tales como dos rupturas uterinas en una casuística de 31 casos $(6.4 \%)$ que consideramos alto. Desde ese año se optó la conducta de practicar cesárea en todos los casos de antecedentes de cesárea, se tuvo en cuenta además el cambio de mentalidad de nuestras mujeres en relación con limitar el número de hijos y a una razón de carácter ético; de no fomentar conductas diferentes en el manejo de pacientes institucionales $y$ particulares (6).

D. En el año 1981 en el trabajo de Análisis de la Indicación Cesárea en el Hospital San Ignacio 1978-1981 (17), se encontró, que los factores que más habían contribuido a su incremento, habían sido en orden descendente:

Distocia

Cesárea anterior

Presentación de Pelvis

Sufrimiento Fetal

Este hallazgo coincide con las publicaciones revisadas $(17,21,24)$.

E. La monitoria fetal, superada la etapa de aprendizaje, ha permitido detectar tempranamente aquellos casos que se benefician de la cesárea.

F. Coincidiendo con los hallazgos de muchos autores, hemos adoptado la conducta de aumentar cada vez más el uso de la cesárea en aquellos casos que por diferentes razones se debe suspender el embarazo y el feto tanto clínica como ecográficamente se considera pequeno $(1,14,21,24)$.

Todos estos cambios que consideramos altamente benéficos, son el resultado de las evaluaciones periódicas de las normas y la consulta permanente de la literatura.

\section{MATERIAL Y METODOS}

En el presente trabajo se estudian las pacientes a quienes se les atendió el parto o cesárea, en el Hospital San Ignacio durante los años 1983 y 1984. Esa casuística está compuesta en un $87 \%$ por pacientes del Seguro Social. El resto corresponde a pacientes de Alto Riesgo referidas de! Centro de Salud del PAMI y un número muy pequeño de pacientes institucionales.

Durante los dos años se atendió un gran total de 6.645 casos obstétricos discriminados en partos vaginales, cesáreas y partos instrumentados (ver Tabla No. 1).

\begin{tabular}{|c|c|c|c|c|}
\hline $\begin{array}{r}\text { HOS } \\
\text { MUESTRA }\end{array}$ & $\begin{array}{l}\text { TA } \\
\text { ESA } \\
\text { ITA } \\
\text { DE }\end{array}$ & $\begin{array}{l}\text { A No } \\
\text { A EN } \\
\text { AN I } \\
\text { UDI }\end{array}$ & $\begin{array}{l}\mathrm{ACI} \\
983\end{array}$ & 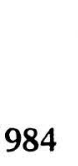 \\
\hline & & EMDIDOS & & TUDINDOS \\
\hline & мо. & $z$ & no. & 2 \\
\hline TOTAL CASOS & 6.565 & & 1.400 & \\
\hline PARTOS ESPONTANEOS & 5,383 & 81.9 & 1.200 & 22.3 \\
\hline CESAREAS & 1.024 & 15.4 & 200 & 19.6 \\
\hline PARTOS VAG. INST. & 158 & 2.4 & & \\
\hline
\end{tabular}

Para el estudio se tomó una muestra representativa y proporcional de cesáreas y partos vaginales espontáneos; doscientos y mil doscientos respectivamente $y$ en forma sistemática, se tomaron los casos de estudio, uno de cada cinco. 
De la historia clínica se tomaron las siguientes variables: edad, paridad, edad gestacional, complicaciones prenatales, presentación, número de fetos por embarazo, indicación de la cesárea, peso al nacer, Apgar al minuto y a los cinco minutos, complicaciones intraoperatorias de la cesárea, complicaciones postoperatorias, complicaciones del puerperio de los partos, muertes perinatales, muertes maternas y estancia hospitalaria; la morbilidad neonatal precoz se descartó por dificultad en obtener la información completa y la falta de seguimiento.

Para el análisis estadístico se usó la prueba $X^{2}$ con un nivel de significancia de $P<0.05$.

\section{RESULTADOS}

\section{Edad materna}

No se encontró diferencia significativa, con relación a estas variables, (ver Tabla No. 2).

TABLA No. 2

CESAREA EN EL

HOSPITAL SAN IGNACIO

DISTRIBUCION EDAD MATERNA

\begin{tabular}{lrrrr}
\hline \multirow{2}{*}{ EDAD } & \multicolumn{2}{c}{ CESAREAS } & \multicolumn{2}{c}{ PARTOS } \\
\hline 17 Y MENOS & 3 & 1.5 & 47 & $3.9 *$ \\
18 A 34 & 177 & 88.5 & 1.067 & $39.0 *$ \\
35 Y MAS & 20 & 10.0 & 86 & $7.1 *$ \\
TOTAL & 200 & & 1.200 &
\end{tabular}

- no significativa

En términos generales se puede decir que se trata de una población joven en donde los grupos extremos o de mayor riesgo representan una casuística baja, de 23 casos o un (11.5\%) para la cesárea y de 133 casos $(11 \%)$ para el grupo de parto.

\section{OBJETIVOS.}

\section{Objetivo General}

Determinar las características de las pacientes a quienes se practicó cesárea con relación a variables demográficas y de salud y compararlas con un grupo proporcional de partos vaginales espontáneos, atendidos en el mismo período 1983 y 1984.

\section{Obje tivos específicos}

Determinar si las indicaciones de cesárea correspondieron a las normas del departamento $y$ evaluar los resultados en términos de morbilidad materna y mortalidad perinatal.

Comparar la morbilidad prenatal y post-parto de los dos grupos.

Estudiar el efecto de la cesárea y el parto en la estancia hospitalaria.

\section{Gravidez}

Con relación a esta variable se encontró una diferencia significativa de un mayor porcentaje de pacientes nulíparas en el

\section{TABLA No. 3 CESAREA EN EL HOSPITAL SAN IGNACIO DISTRIBUCION POR GRAVIDEZ}

\begin{tabular}{|c|c|c|c|c|}
\hline & \multicolumn{2}{|c|}{ CESAREAS } & \multicolumn{2}{|c|}{ PARTOS } \\
\hline & No. & 7 & No. & $z$ \\
\hline 0 & 106 & 53.0 & 462 & $38.5^{\circ}$ \\
\hline 1 A 4 & 85 & 42.0 & 699 & $58.2^{\circ}$ \\
\hline 5 Y MAS & 9 & 4.5 & 39 & $3.3^{* *}$ \\
\hline TOTAL & 200 & 100.0 & 1200 & 100.0 \\
\hline
\end{tabular}

- significativa

$\because$ no significativa 
grupo de cesárea $(53 \%)$ y de $38.5 \%$ en el de partos. Este hallazgo fue de gran importancia en relación con complicaciones del embarazo e indicación de cesárea.

Llama la atención el reducido número de grandes multíparas nueve casos $(4.5 \%)$ en cesárea y treinta y nueve $(3.3 \%)$ en partos. No hubo diferencia significativa. (ver Tabla No. 3).

TABLA No. 4 CESAREA EN EL HOSPITAL SAN IGNACIO DISTRIBUCION POR EDAD GESTACIONAL $1983-1984$

\begin{tabular}{|c|c|c|c|c|c|}
\hline \multirow[b]{2}{*}{ SEMANAS } & \multicolumn{2}{|c|}{ CESAREA } & \multicolumn{2}{|c|}{ PARTOS } & \\
\hline & No. & 7 & no. & 7 & \\
\hline MAS DE 42 & 3 & 1.5 & 15 & 1.2 & \\
\hline 38 A 42 & 151 & 75.8 & 1.046 & 87.1 & \\
\hline SUBTOTAL & 154 & 77.0 & 1.061 & 88.3 & - \\
\hline 33 A 37 & 39 & 19.5 & 116 & 9.6 & \\
\hline MENOS DE 33 & 7 & 3.5 & 23 & 1.9 & \\
\hline SUBTOTAL & 46 & 23.0 & 139 & 11.5 & - \\
\hline
\end{tabular}

\section{EDAD GESTACIONAL}

El porcentaje de embarazos pretérmino, por debajo de treinta y ocho semanas, fuc de cuarenta y seis casos, $23 \%$ para el grupo de cesáreas contra ciento treinta y nueve, $11.5 \%$ para el grupo de partos, con una diferencia altamente significativa.

En las Tablas No. 5 y 6 , se muestra la correlación que tuvo la prematurez con la mortalidad perinatal, en el grupo de cesárea seis casos $75 \%$ y en el de partos 11 casos $50 \%$.

El número de muertes perinatales de embarazos muy tempranos fue alto en los
TABLA No. 5

CESAREA EN EL HOSPITAL SAN IGNACIO MORTALIDAD PRETERMINO CESAREA

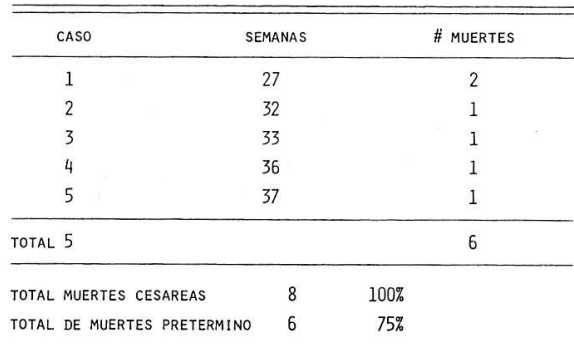

TABLA No. 6 CESAREA EN EL HOSPITAL SAN IGNACIO MORTALIDAD PRE-TERMINO PARTOS

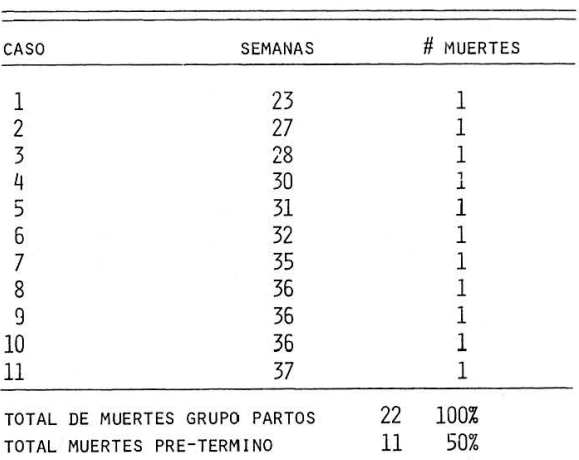

dos grupos. Cuatro, $50 \%$ en cesárea y siete, $31.8 \%$ en el grupo de partos.

No se encontraron clínicamente casos de post-término en los dos grupos.

\section{COMPLICACIONES PRENATALES $Y$ PRESENTACIONES ANORMALES}

En el grupo de cesárea, las complicaciones médicas prenatales fueron numérica- 
mente y en severidad, mayores que en el grupo de partos. Noventa y dos casos, $46 \%$ y ciento sesenta y cinco, $14.5 \%$ en el grupo de partos, lo que concuerda con varias publicaciones $(12,15,17,21)$. (Tabla No. 7).

Las complicaciones prenatales en los dos grupos y en orden de frecuencia fueron: ruptura prematura de membranas, cesárea 34 casos, (17\%); partos 86 casos $(7.1 \%$ ) predominando los casos más graves en cesárea.

Las complicaciones hipertensivas ocuparon el segundo lugar, cesárea 25 casos $(17 \%)$ y partos 58 casos, $(4.8 \%)$. La distribución de las diferentes modalidades, leve, severa, etc. se presenta en la Tabla No. 7.

El resto de complicaciones correspondieron a amenaza de aborto y parto prematuro, infección urinaria y otros (ver Tabla No. 7).

TABLA No. 7

CESAREA EN EL HOSPITAL SAN IGNACIO COMPLICACIONES PRENATALES

\begin{tabular}{|c|c|c|c|c|}
\hline & \multicolumn{2}{|c|}{ CESAREAS } & \multicolumn{2}{|c|}{ PARTOS } \\
\hline & No. & 6 & No. & 7 \\
\hline R,P,M. & 16 & 8.0 & 64 & 5.3 \\
\hline R.P,M, PROLONG, & 13 & 6.5 & 22 & 1.8 \\
\hline AMEIIONITIS (R.P.M.) & 5 & 2.5 & -- & -- \\
\hline SUBTOTAL & 34 & 17.0 & 86 & 7.1 \\
\hline PRE ECLNAP LEVE & 6 & 3.0 & 34 & 2.8 \\
\hline PRE ECLAYIP GRAVE & 9 & 4.5 & 10 & 0.8 \\
\hline ECLAPEPSIK & 2 & 1.0 & - & - \\
\hline E,V,H,C. & 5 & 2.5 & 10 & 0.8 \\
\hline EVHC + PREECLAMP & 3 & 1.5 & 4 & 0.3 \\
\hline SUBTOTAL & 25 & 12.5 & 58 & $4.8^{*}$ \\
\hline PLACERTA PREVIA & 3 & 1.5 & 2 & 0.1 \\
\hline $\begin{array}{l}\text { AMENAZA PARTO } \\
\text { PREM Y ABORTO }\end{array}$ & 11 & 5.5 & 1 & 0.08 \\
\hline IMFECC. URIN. & 10 & 5.0 & 15 & 1.25 \\
\hline PREDIATETES & 2 & 1.0 & -- & $-\cdots$ \\
\hline VARIOS & 7 & 3.5 & 13 & 1.08 \\
\hline TOTAL & 92 & 46.0 & 175 & $14.50^{*}$ \\
\hline
\end{tabular}

* significativa

\section{DISTRIBUCION POR PRESENTACION}

Là presentación de pelvis fue significativamente mayor en el grupo de cesárea, veinticinco casos $(12.5 \%)$ y solamente trece casos, $(1.1 \%)$ en los partos. (Tabla No. 8). Este hallazgo se relacionó en forma importante con la indicación de cesárea como se verá posteriormente.

TABLA No. 8

CESAREA EN EL HOSPITAL SAN IGNACIO DISTRIBUCION POR PRESENTACION

\begin{tabular}{|c|c|c|c|c|}
\hline & \multicolumn{2}{|c|}{ CESAREA } & \multicolumn{2}{|c|}{ PARTOS } \\
\hline & No. & 3 & No. & 8 \\
\hline VERTICE & 153 & 79 & 1.187 & $98.9^{*}$ \\
\hline PELVIS & 25 & 12.5 & 13 & $1.1^{*}$ \\
\hline SIT. TRANSV. & 17 & 8.5 & $\cdots$ & --- \\
\hline TOTAL & 200 & 100 & 1.200 & 100 \\
\hline
\end{tabular}

Las situaciones transversas diagnosticadas al final del embarazo, incluyendo los embarazos gemelares, fueron 17 casos $(8.5 \%)$ y correspondieron al grupo de cesárea.

\section{EMBARAZOS MULTIPLES}

Los embarazos gemelares fueron significativamente más frecuentes en cesárea, diez casos $5 \%$ y ocho $0.66 \%$ en los partos. Esta situación influyó en forma importante, en la indicación de cesárea (Tabla No. 8A).

TABLA No. 8A

CESAREA EN EL

HOSPITAL SAN IGNACIO

EMBARAZOS MULTIPLES

\begin{tabular}{lrrrr}
\hline & CESAREA & $\%$ & PARTOS \% \\
\hline CASOS & 200 & 100. & 1200 & 100. \\
GEMELARES & 10 & 5. & 8 & 0.66
\end{tabular}




\section{INDICACION DE LA CESAREA}

En orden descendente de frecuencia, la primera indicación de cesárea fue la desproporción cefalo pélvica, cincuenta y siete casos, $28.5 \%$; en segundo lugar el antecedente de una o más cesáreas con cuarenta y un casos, $25 \%$. En ningún caso de antecedente de cesárea se le permitió parto por vía vaginal, siguiendo la norma.

Por ruptura prematura de membranas se operaron veintidós casos, que representaron un $11 \%$ y en cuarto lugar por sufrimiento fetal agudo intraparto, diez y seis casos, $8 \%$; las presentaciones de pelvis quince casos $7.5 \%$. Estos hallazgos son casi idéntícos a los encontrados en las casuísticas norteamericanas $(12,17,21,24)$. (Tabla No. 9).

\section{TABLA No. 9 \\ CESAREA EN EL \\ HOSPITAL SAN IGNACIO (INDICACION)}

\begin{tabular}{lrr}
\hline \hline INDICACION & NO. & 2 \\
\hline OSEAS & & \\
DESPROPORCION CEFALOPELVICA & 57 & 28.5 \\
FETALES & 15 & 7.5 \\
PRESENTACION DE PELVIS & 5 & 2.5 \\
SITUACION TRANSVESA & 8 & 3.5 \\
EMBARAZO GEMELAR & 16 & 8.0 \\
SUFRIMIENTO FETAL AGUDO & & \\
OVULARES & 22 & 11.0 \\
RUPTURA PREMATURA DE MEMBRANAS & 6 & 3.0 \\
PLACENTA PREVIA & 6 & 3.0 \\
ABRUPTIO PLACENTAE & & \\
DINANICAS & 8 & 0.5 \\
DISTOCIA DE CONTRACCION & & \\
OTRAS & 41 & 25.0 \\
ANTECEDENTE DE CESAREA & 6 & 3.0 \\
PRE-ECLAMPSIA GRAVE & 3 & 1.5 \\
ECLAMPSIA & 7 & 2.0 \\
VARIAS & & \\
\hline TOTAL & 200 & 100. \\
\hline
\end{tabular}

La indicación por placenta previa, seis casos y abruptio seis casos (diagnósticos intraparto), representaron solamente el $6 \%$, pero correspondió a la patología de mayor riesgo materno fetal, hasta el punto que fue necesario practicar histerecto- mía en dos casos por apoplegia ú tero placentárea y el otro por una pelviperitonitis muy severa.

Dos muertes fetales del grupo de cesárea, correspondieron a un embarazo gemelar de 27 semanas con placenta central total.

Se operaron nueve casos, por complicaciones hipertensivas graves $(4.5 \%)$. Los neonatos de estos casos nacieron con muy buena calificación de Apgar. No se presentaron muertes y la conducta correspondió a la norma respectiva. El detalle del resto de indicaciones se presenta en la Tabla No. 9.

\section{COMPLICACIONES INTRAOPERATORIAS}

En los doscientos casos de cesárea, se presentaron complicaciones intra-operatorias en diez y siete casos para un $9.5 \% \mathrm{cu}$ yo detalle se presenta en la Tabla No. 10.

TABLA No. 10

CESAREA EN EL

HOSPITAL SAN IGNACIO

COMPLICACIONES

INTRA-OPERATORIAS

\begin{tabular}{lrr}
\hline & NO. & $\%$ \\
\hline HEMORRAGIA & 10 & 58.8 \\
EXTRACCION DIFICIL & 4 & 23.5 \\
DESGARRO DEL SEGMENTO & 2 & 11.7 \\
CESAREA HISTERECTOMIA & 1 & 5.8 \\
TOTAL & 17 & 100.0 \\
\hline
\end{tabular}

$E_{i}$ las complicaciones intra-operatorias predominaron la hemorragia y los desgarros. A estas complicaciones relacionadas con el acto quirúrgico en sí, se suma la patología del embarazo que determinó la indicación de cesárea, circunstancia que 
explica la mayor morbimortalidad materna y perinatal de la cesárea en comparación con el parto vaginal espontáneo $(5,21,24)$.

\section{COMPLICACIONES POST-CESAREA Y PUERPERIO}

Las complicaciones post-operatorias de cesárea, se presentaron en treinta y tres casos, $16 \%$ del total de cesáreas, con un promedio franco de infección incluida una pelviperitonitis que requirió reintervención con histerectomía (Tabla No. 11).

\section{TABLA No. 11 \\ CESAREA EN EL \\ HOSPITAL SAN IGNACIO \\ COMPLICACIONES \\ POST-OPERATORIAS Y POST-PARTO}

\begin{tabular}{lrccc}
\hline \hline & \multicolumn{2}{c}{ CESAREAS } & \multicolumn{2}{c}{ PARTOS } \\
& NO, & $\%$ & NO. & $\%$ \\
\hline ENDOMETRITIS & 13 & 38.3 & 9 & 30. \\
PORT-OP FEBRIL & 10 & 30.3 & 1 & 3.3 \\
INFECCION HERIDA & 4 & 12.1 & - & - \\
PELVIPERITONITIS & 1 & 3.3 & - & - \\
INFECCION URINARIA & 3 & 9.0 & 2 & 6.6 \\
ECLAMPSIA & 1 & 3.3 & - & - \\
CEFALEA ANESTESICA & 1 & 3.3 & - & - \\
ENF PELV INFLAM. & - & --- & 1 & 3.3 \\
HEMORRAGIA & - & --- & 9 & 30. \\
ATONIA UTERINA & - & --- & 5 & 16.6 \\
HEMATOMA PERINEAL & - & --- & 1 & 3.3 \\
RETENCION DE PLACENTA & - & --- & 1 & 3.3 \\
INVERSION UTERINA & - & --- & 1 & 3.3 \\
TOTAL & 33 & 100.0 & 30 & 100. \\
\hline
\end{tabular}

* significativa

Las complicaciones post-parto fueron solamente treinta casos o sea un $2.3 \%$. Su severidad fue mucho menor que la de la cesárea, predominando la infección y en segundo lugar la hemorragia.

Al seleccianar la cesárea como conducta obstétrica versus el parto vaginal, se debe tener siempre en mente su mayor morbilidad y riesgos de muerte materna.

\section{MORTALIDAD MATERNA}

En ninguno de los grupos se presentó mortalidad materna, gracias a que las complicaciones se pudieron controlar satisfactoriamente. Este hallazgo se puede considerar como una evaluación muy buena de la calidad de la atención hospitalaria.

\section{DISTRIBUCION DE PESO AL NACER}

Como se puede observar se encontró un mayor número de fetos de bajo peso, menores de 2.500 gramos, en el grupo de cesáreas: cuarenta y tres casos $(20.4 \%)$, por 121 casos $(10 \%)$ en el de partos, diferencia estadísticamente significativa. (Tabla No. 12).

\section{TABLA No. 12 \\ CESAREA EN EL \\ HOSPITAL SAN IGNACIO \\ DISTRIBUCION POR PESO \\ DEL RECIEN NACIDO}

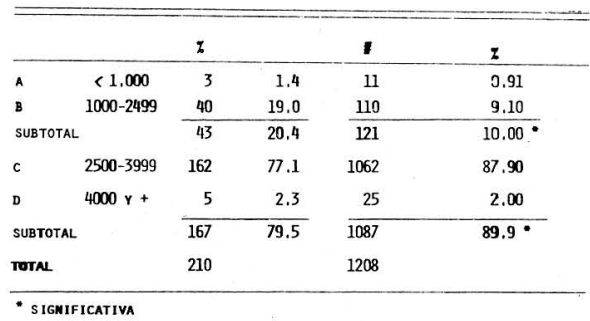

El bajo peso fue el factor que más se correlacionó como causa de muerte perinatal en los dos grupos así: de las ocho muertes en cesárea seis correspondían a fetos menores de 2.500 gramos y de veintidós muertes de parto, trece correspondieron a fetos de bajo peso.

\section{CALIFICACION DE APGAR}

Es método de evaluación final de la atención. Es cuestionado por muchos, pero definitivamente refleja por lo menos en parte la buena o mala conducta. 
EI análisis comparativo de la calificación del Apgar al minuto y a los cinco minutos, muestra muy claramente la diferencia significativa, de un mayor número de calificaciones bajas en el grupo de cesárea. Esto es muy explicable debido a la mayor patología de los embarazos, al mayor número de embarazos pretérmino y con bajo peso o con retardo del crecimiento y a los efectos de la anestesia; pero es muy claro que los fetos se recuperan y el porcentaje de Apgar alto a los cinco minutos es muy satisfactorio. ( $\mathrm{Td}$ blas 13 y 14).

TABLA No. 13

CESAREA EN EL

HOSPITAL SAN IGNACIO

CALIFICACION DE

APGAR AL MINUTO

\begin{tabular}{lrrrr}
\hline & \multicolumn{2}{c}{ CESAREa } & \multicolumn{2}{c}{ PARTOS } \\
\hline 0 & 7 & 3.3 & 15 & 1.2 \\
1 A 6 & 31 & 14.7 & 53 & $4.3^{*}$ \\
7 A 10 & 172 & 81.9 & 1140 & $94,4^{*}$ \\
TOTAL & 210 & & 1208 & \\
\hline
\end{tabular}

"sigmificativa

TABLA No. 14

CESAREA EN EL

HOSPITAL SAN IGNACIO

CALIFICACION DE

APGAR A LOS 5 MINUTOS

\begin{tabular}{lrrrr}
\hline & \multicolumn{2}{c}{ CESAREA } & \multicolumn{2}{c}{ PARTOS } \\
& No. & \multicolumn{2}{c}{$\%$} & \multicolumn{2}{c}{ No. } & \multicolumn{1}{c}{} \\
\hline & 7 & 3.3 & 18 & 1.5 \\
1 A 6 & 8 & 3.8 & 8 & $0.6^{*}$ \\
7 A 10 & 195 & 92.9 & 1182 & $97.8^{*}$ \\
TOTAL & 210 & & 1208 & \\
\hline
\end{tabular}

"SIgN IF ICAT IVA
Nuestros resultados coinciden con la mayoría de los hallazgos de la literatura obstétrica $(4,15,21,24)$.

\section{MORTALIDAD PERINATAL}

En la mortalidad perinatal, solamente se incluyerón como muertes neonatales, las que se presentaron en nuestra Unidad de recién nacidos, por tanto en la gran mayoría no se incluyeron los siete días de muerte neonatal precoz por la dificultad de seguimiento.

En el grupo de cesárea, se presentaron 8 muertes para una tasa de mortalidad de $38 \%$ y en los partos 22 muertes con una tasa de $18.2 \%$ (Tabla 15).

TABLA No. 15

CESAREA EN EL HOSPITAL SAN IGNACIO MORTALIDAD PERINATAL (TASA \% )

\begin{tabular}{lrrrr}
\hline \hline & \multicolumn{3}{c}{ CESAREAS } & \multicolumn{3}{c}{ PARTOS } \\
& NO. & $\%$ NO. & \multicolumn{1}{c}{$\%$} \\
\hline RECIEN NACIDOS & 210 & & 1208 \\
MORT PERINATAL TOTAL & 8 & 38.0 & 22 & 18.2 \\
ANTEPARTO & 6 & 28.5 & 14 & 10.7 \\
INTRAPARTO & 1 & 4.7 & 2 & 1.6 \\
NEONATAL & 1 & 4.7 & 6 & 5.7 \\
& & & & \\
\hline
\end{tabular}

Con relación al tiempo en que se presentaron las muertes, en cesárea 6, (75\%) fueron anteparto y 14 casos $(63.6 \%)$ en el grupo de parto.

Las muertes intraparto neonatal, fueron solamente, en cesárea dos casos y ocho en el grupo de partos, (Tabla No. 16).

Los dos casos de cesárea correspondían a dos fetos con malformaciones congénitas: anencefalia y microcefalia, labio leporino y toxoplasmosis congénita respectivamente. 
TABLA No. 16

CESAREA EN EL

HOSPITAL SAN IGNACIO

MODALIDAD DE MUERTE

\begin{tabular}{lcccc}
\hline & \multicolumn{2}{c}{ CESAREA } & \multicolumn{2}{c}{ PARTOS } \\
& No. & $\%$ & No. & $\%$ \\
\hline ANTEPARTO & 6 & 75 & 14 & 63.6 \\
INTRAPARTO & 1 & 12.5 & 2 & 9.09 \\
NEONATAL & 1 & 12.5 & 6 & 27.27 \\
TOTAL & 8 & 100.0 & 22 & 100.0 \\
\hline
\end{tabular}

En el grupo de parto las causas de las muertes intraparto fueron: sífilis congénita y un caso de microcefalia.

Las seis muertes neonatales $27.2 \%$, correspondieron a casos pretérmino de 23 a 30 semanas. Estos hallazgos corresponden a varias publicaciones $(10,21,24,25)$.

La ruptura prematura de membranas se presentó en cuatro de las muertes de cesárea y solamente en dos casos del grupo de partos y en embarazos de 23 a 28 semanas respectivamente.

Las muertes pretérmino en cesárea fueron el $75 \%$ y $50 \%$ en el grupo de parto.

Los casos de muerte de fetos de menos de 2.500 gramos fueron 6 y 13 en cesárea

TABLA No. 17

CESAREA EN EL HOSPITAL SAN IGNACIO MORTALIDAD PERINATAL SEGUN PESO

(TASA \%)

\begin{tabular}{lcrrrrr}
\hline PESO & \multicolumn{3}{c}{ CESAREA } & \multicolumn{3}{c}{ PARTO } \\
& \# R.N. & \# MORT \%. & \# R.N. & \# MORT. \% \\
\hline$<2500$ & 43 & 6 & 139.8 & 121 & 13 & $107.4^{*}$ \\
$\Rightarrow 2500$ & 167 & 2 & 11.9 & 1087 & 9 & $8.3^{*}$ \\
POTAL & 210 & 8 & 38.0 & 1208 & 22 & 18.2 \\
\hline
\end{tabular}

y parto respectivamente $y$ de 2 y 9 casos de fetos de más de 2.500 gramos (Tabla No. 17).

Con relación a mortalidad según peso, las muertes de fetos de menos de 2.500 gramos fue de seis casos en cesárea $(75 \%)$ $y$ trece muer tes en el grupo de partos (59\%).

Las muertes de más de 2.500 gramos fueron solamente para cesárea dos casos y para partos 9 casos.

\section{ESTANCIA HOSPITALARIA}

El promedio de estancia hospitalarid en el grupo de cesárea fue de 3.38 días y de 1.56 para el de partos. Solamente se tuvo en cuenta la hospitalización desde el día del parto hasta que se dio de alta sin incluir hospitalizaciones durante el embarazo por complicaciones del mismo. Consideramos este resultado muy satisfactorio.

\section{MORTALIDAD MATERNA}

En la muestra de nuestro estudio de 1.400 casos, no se encontró ninguna muerte materna y corresponde a los años 1983-1984.

\section{COMENTARIOS}

La preocupación general ante el gran incremento que ha tenido la cesárea y la dificultad de cuantificar cuál ha sido el beneficio para la madre y el hijo, en términos de disminuir la morbimortalidad, representa una incógnita difícil de aclarar por los resultados opuestos que se encuentran en la literatura $(15,17,21,24)$.

Se sabe que las causas principales de ese incremento se relacionan con el cambio y actualización de normas en el manejo de la distocia, el antecedente de cesá- 
rea, el sufrimiento fetal, la presentación de pelvis y el embarazo múltiple y el feto de bajo peso.

Teniendo en cuenta las características socio-económicas bajas de nuestra población y las demográficas y médicas de las pacientes de cesárea halladas en el estudio, el incremento de la cesárea a un $15.3 \%$ lo considero lógico y con resultados que demuestran la buena calidad de la atención médica.

Como un resultado lógico la morbimortalidad perinatal y la morbilidad materna, fue significativamente mayor y más severa en el grupo de cesárea y coincide con varias publicaciones $(5,10,12,15,21,25)$.

En nuestros resultados, llama la atención la al ta frecuencia y las repercusiones de la ruptura de membranas, patología que representa una prioridad en el estudio de sus posibles causas, prevención y manejo.

Con relación a otras complicaciones prenatales, el resultado de una conducta quirúrgica precoz en los casos severos de complicaciones hipertensivas fue muy satisfactorio, pues no se presentaron muertes ni morbilidad inmediata de los neonatos ni de las madres.

Las complicaciones placentarias, mostraron una vez más el alto riesgo que representan para la madre y el hijo y la necesidad de asegurar en los cursos de postgrado que se adquiera un buen criterio médico y entrenamiento quirúrgico para su correcto manejo.

Uno de los objetivos del presente trabajo, era el determinar hasta qué punto, se habían cumplido las normas del departamento y evaluar los resultados. Los comentarios al respecto se pueden resumir así: en los casos de distocia se prefirió la cesárea y no se usaron fórceps medios. Cumpliéndose la norma, solamente se aplicaron fórceps bajos y de desprendimiento $(15,19,21,25)$. En los casos de distocia en que se practicó cesárea, no hubo muertes fetales y los Apgares fueron excelentes.

En los casos de antecedente de cesárea, se practicó cesárea en el $100 \%$ según la norma y los resultados neonatales y maternos fueron excelentes. Considero que en nuestro medio las instituciones, en general, no tienen la disponibilidad de recursos en forma permanente para darle a los casos seleccionados prueba de trabajo de parto vaginal como lo sostienen varios trabajos previos $(6,21,24)$.

No se encontraron en nuestra casuística dehiscencias o rupturas uterinas de la cicatriz previa.

En ruptura prematura de membranas nuestra norma es practicar cesárea, cuando las condiciones de inducción no son favorables y falla la inducción. Tuvimos 22 casos que se operaron. En 20, los neonatos nacieron y evolucionaron satisfactoriamente. Se presentó una muerte neonatal por toxoplasmosis y microcefalia y se operó una paciente con feto muerto y amnionitis que no respondió a varios intentos de inducción.

En sufrimiento fetal agudo, optamos por mantener la paciente en decúbito lateral, dar oxígeno para recuperar el niño in útero y si se confirma el sufrimiento con monitoria externa, se practica cesárea.

Se operaron 16 casos y no se presentaron muertes intra-cesárea o neonatales.

Una delasindicaciones de más controversia en cuanto al beneficio de la cesárea, es la presentación de pelvis $(5,7,8,20,21,22,23$, 25); pero no hay duda que esta presentación anormal, tiene un porcentaje importante de morbimortalidad perinatal. Apdrentemente los fetos que menos se benefician de la cesárea, son los fetos a término, 
de peso adecuado y en pelvis completa. Hay datos muy contradictorios con relación a niños pequeños y de bajo peso (4, $5,7,8,15,20,21,22,23,25)$.

Nuestra norma en presentación de pelvis es practicar cesárea en todas las pacientes primigestantes y en los embarazos pretérmino con niños pequeños. En las multigestantes con buenos antecedentes $y$ evaluación clínica favorable, permitir prueba de trabajo. Si el progreso no es rápido en cuanto dilatación y descenso de la presentación practicar cesárea. En los 15 casos operados el resultado fue excelente. No se cumplió la norma en 3 casos de primigestantes a quienes se permitió parto vaginal asistido, con feto en buenas condiciones.

Consideramos el embarazo gemelar de alto riesgo para el parto vaginal. Nuestra norma considera que en los casos en que los dos fetos estén en presentación vértice se puede dejar evolucionar el parto vaginal; en el resto practicar cesárea. Con relación al manejo del embarazo gemelar existe gran controversia $(3,15,21,24)$.

En nuestra casuística se operaron 8 casos, de acuerdo con la norma, con buenos resultados. No se cumplió en dos casos. Uno con primer feto en pelvis y el segundo en pelvis y otro con fetos en pelvis y transversa. No se presentó morbimortalidad. Es necesario investigar más al respecto, pero de todas maneras la tendencia es practicar la cesárea en embarazos múltiples.

Nosotros consideramos que la cesárea beneficia considerablemente a niños pequeños por prematurez o retardo de crecimiento. La literatura presenta resultados contradictorios $(14,15,21,24)$.

La mortalidad perinatal en nuestro estudio: En cesáred y parto, 38.\% y $18.2 \%$ y total de $21.1 \%$ la considero muy satisfactoria y un buen parámetro en favor de una buena calidad de atención hospitalaria, fue menor que la encontrada en el estudio cooperativo del CLAP $36.1 \%$ 1980 (18), y muy similar a la encontrada por Germán Uriza en el trabajo cooperativo institucional, tasa de $21.03 \%(25)$.

Los factores que más contribuyeron a la mortalidad perinatal fueron: el bajo peso al nacer, la prematurez, el retardo de crecimiento intrauterino y las complicaciones prenatales especialmente la ruptura prematura de membranas, los embarazos gemelares y las malformaciones congénitas, especialmente la anencefalia. Estos hallazgos coinciden con los reportados en la literatura.

Si se analiza que un número muy importante de las muertes en los dos grupos $75 \%$ y $63 \%$ se presentaron antes de ingresar al hospital, se puede deducir que el manejo y las conductas en la atención hospitalaria fue de excelente calidad.

En la actualidad se están usando rutinariamente antibióticos profilácticos en cesárea y se ha comprobado los buenos resultados. Para una detección precoz de la distocia se está implantando el uso del partograma y como plan de trabajo nos hemos fijado la evaluación minuciosa de las normas.

Finalmente se puede comentar que la diferencia encontrada de un mayor riesgo del grupo de cesárea en comparación al grupo de parto vaginal espontáneo, coincide con las diferentes publicaciones (5).

El hecho de haber cumplido las normas en la gran mayoría de los casos, excepto en unos pocos casos de presentación de pelvis y embarazos gemelares; el no haber tenido muertes maternas en la muestra estudiada, el haber tenido unas tasas de mortalidad de $38 \%$ en la cesárea, de $18.2 \%$ en los partos y una tasa de mortalidad total de 21.1 son resultados muy satisfactorios y una evaluación muy posiţiva del funcionamiento de nuestro departamento. 


\section{RESUMEN}

En el presente trabajo se analiza la evolución de la cesárea en el período 1975 a 1984 con un incremento de $3.1 \%$ a $15.3 \%$ debido a los cambios en las diferentes normas de manejo. Los factores que más influyeron en el incremento de cesárea fueron en orden de frecuencia: distocia, antecedente de cesárea y presentación de pelvis.

Se estudiaron las características de los casos de cesárea con relación a variables demográficas y médicas y se comparan con un grupo de partos espontáneos. La muestra tomada en forma sistemática fue de 200 cesáreas y 1.200 partos espontáneos.

Los resultados del estudio fueron: en cuanto a edad materna los grupos resultaron comparables; en cambio el resto de variables fueron significativamente desfavorable para el grupo de cesárea así: en cuanto a gravidez, el número de primigestantes fue de $53 \%$ y $38.5 \%$; el número de partos pretérminos $23 \%$ y $11.5 \%$; las complicaciones prenatales fueron numéricamente y en severidad mayor en la cesárea $46 \%$ y $14.5 \%$. En orden descendente fueron: ruptura prematura de membranas $17 \%$ y $7.1 \%$, complicaciones hipertensivas $12.5 \%$ y $4.87 \%$, amenaza de aborto y parto prematuro $5.5 \%$ y $0.08 \%$, infección urinaria $5 \%$ y $1.25 \%$, placenta previa $1.5 \%$ y $0.1 \%$.

Las presentaciones anormales; pelvis 12.5 y $1.1 \%$. En cesárea se presentaron 17 casos de situación transversa $(8.5 \%)$. Los embarazos gemelares $5 \%$ y $0.7 \%$

En relación con la indicación de cesárea los hallazgos fueron: desproporción cefa-

* Profesor titular. Director del Departamento Ginecología y Obstetricia, Pontificia Universidad Javeriana. lopélvica $28.5 \%$; antecedentes de cesárea $25 \%$; ruptura prematura de membrana $11 \%$; sufrimien to fetal agu do $8 \%$; presentación de pelvis $7.5 \%$, placenta previa $6 \%$ y complicaciones hipertensivas $4.5 \%$.

Las complicaciones intraoperatorias se presentaron en un $9.5 \%$ con predominio de la hemorragia.

La morbilidad post-cesárea y puerperio fue significativamente mayor en la cesárea: $16 \%$ contra solamente $2.5 \%$ en partos; predominando la endometritis, $6.5 \%$ y $0.75 \%$. En el grupo de cesárea se practicaron dos histerectomías por apoplegía útero placentaria y peritonitis generalizada.

No se encontró mortalidad materna.

El bajo peso por prematurez y retardo del crecimiento intrauterino fue significativamente mayor en cesárea: $20.4 \%$ y $10 \%$.

El Apgar al minuto fue más bajo en la cesárea: de 6 y menos, $14.8 \%$ y $4.3 \%$ y a los cinco minutos: $3.8 \%$ y $0.6 \%$ en partos.

La mortalidad perinatal en cesárea fue $38 \%$ y $18.2 \%$ en los partos y la mortalidad total de $21.1 \%$. Las principales causas de esa mortalidad fueron: prematurez y bajo peso, ruptura de membranas y complicaciones placentarias. La mortalidad anteparto fue de $75 \%$ y $14 \%$ respectivamente, neonatales $12.5 \%$ y $27.4 \%$ en partos.

El estudio mostró que las normas del Departamento se cumplieron correctamente excepto en algunos casos de embarazo gemelar y presentación de pelvis.

La estancia hospitalaria fue de 3.38 días en cesárea y 1.5 en partos y ninguna muerte materna. 


\section{BIBLIOGRAFIA}

1. BARRET JM; BOEHM FH; VAUGHN WK. The effec of type of delivery on neonatal outcome in singleton infants of birth weight of $1,000 \mathrm{~g}$ or less. JAMA 1983, Aug 5; 250 (5): 625-9.

2. BERNAL ROBERTO. Embarazo gemelar. 1976 - 1978.

3. CHERVENAK FA; JOHNSON RE; YOUCHA S.; HOBBINS JC; BERKOWITZ RL. Intrapartum management of twin gestation. Obstet Gynecol 1985 Jan; 65 (1): 119-24.

CHERVENAK FA; JOHNSON RE; BERKOWITZ RL; GRANNUM P; HOBBINS JC. Is routine cesarean section necessary for vertex-breech and vertex-transverse twin gestation? Am. J. Obstet. Gynecol 1984. Jan 1; $148(1): 1-5$.

4. FABER-NIJHOLT R; HUISJES HJ; TOUWEN BC; FIDLER VJ. Neurological follow-up of 281 children born in breech presentation: a controlled study. Br Med J (Clin Res) 1983 Jan 1; 286 (6358): 9-12.

5. GABERT HA. Current status of cesarean births. Obstet Gynecol Annu 1984; 13: 71-82.

6. GELLMAN E; GOLDSTEIN MS; KAPLAN S; SHAPIRO WJ. Vaginal delivery after cesarean section. Experience in private practice. JAMA 1983 Juan 3; 249 (21): 2935-7.

7. GIMOVSKY ML; WALLACE RL; SCHIFRIN BS; PAUL RH, Randomized management of the nonfrank breech presentation at term: a preliminary report. Am J Obstet Gynecol 1983 May $1 ; 146$ (1): 34-40.

8. HOSANG R; JANMOHAMED N. A comparative analysis of breech deliveries between 1974/1975 and $1979 / 1980$ at the University Hospital of the West Indies. West Indian Med J 1984 Mar: 33 (1): 31-5.
9. LANDESMAN $R$; GRABER EA. Abdom inovaginal delivery: modification of the cesarean section operation to facilitate delivery of the impacted head. Am J Obstet Gynecol 1984 Mar 15; 148 (6): 707-10.

10. MORA PADILLA EDMUNDO. Estudio de la Mortalidad Perinatal en el Hospital San Ignacio, 1981.

1.1. MORGAN H. Cesarean section under epidural analgesia $\mathrm{Br} \mathrm{J}$ Hosp Med 1983 Sep; 30 (3): 179-80, 182-3.

12. PERDOMO RAFAEL. Morbi-mortalidad en el antecedente de cesárea. (H.U.S.I.) 1979.

13. PUOLAKKA J; KAUPPILA A; TUIMALA R; JOUPPILA R.; VUORI J. The effect of parturition on umbilical blood plasma levels of norepinephrine. Obstet Gynecol 1983 Jan; 61 (1): 19-21.

14. ROSEN MG; CHIK L. The association between cesarean birth and outcome in vertex presentation: relative importance of birth weight, Dubowitz scores, and delivery route. Am J Obstet Gynecol 1984 Nov. 15; $150(6): 775-9$.

15. SACHS BP; MCCARTHY BJ; RUBIN G; BURTON A; TERRY J; TYLER CW Jr. Cesarean section. Risk and benefits for mother and fetus. JAMA 1983 Cct. $28 ; 250$ (16): 2157-9.

16. SCHUTTERMAN EB; GRIMES DA. Comparative safety of the low transverse versus the low vertical uterine incision for cesarean delivery of breech infants. Obstet Gynecol 1983 May; 61 (5): 593-7.

17. SUAREZ GERMAN; URDANETA HERNAN. Análisis de las Indicaciones de Cesárea. Hospital San Ignacio. 1978-1981.

18. SCHWARCKZ R; FESCINA R, y colaboradores. Publicación científica CLAP No. Montevideo, 1981. 
19. TRAUB AI; MORROW RJ; RITCHIE JW; DORNAN KJ. A continuing use for Kielland's forceps? $\mathrm{Br}$ J Obstet Gynecolog 1984 Sep; 91 (9): 894-8.

20. VAN EYK EA: HUISJES HJ. Neonatal Mortality and morbidity associated with preterm breech presentation. Eur J. Obstet Gynecol Reprod. Biol 1983 Apr. 15 (1): 17-23.

21. Williams, 17 Edición. Pg. 4.

22. WOO JS; CHAN $\mathrm{Ph}$; GHOSH A; WONG V: MA HK. Term breech delivery is a high caesarean section rate justified? Aust NZ J Obstet Gynecol 1983 Feb.; 23 (1): 25-7.
23. WEINSTEIN D; MARGALIOTH EJ; NAVOT D; MOR-YOSEF S.; Eyal F Neonatal fetal death following cesarean section secondary to hyperextended head in breech presentation. Acta Obstet Gynecol Scand 1983; 62 (6): 629-31.

24. MORTIMER G. ROSEN. Special Communication. NIH consensus Development Statement on Cesarean Childbirth. Task Force Obstetrics and Gynecology. Vol. 57, No. 4, April 12, 1980.

25. URIZA G., BARRAGAN J, Mortalidad Perinatal. Estudio Cooperativo Institucional (Informe Preliminar). Revista Colombiana de Obstetricia y Ginecología. Marzo-Abril/86. Vol. XXXVII, No. 2. 